\title{
Solving optimal reactive power problem by improved variable mesh optimization algorithm
}

\author{
Kanagasabai Lenin \\ Department of EEE, Prasad V. Potluri Siddhartha Institute of Technology, India
}

\begin{tabular}{l}
\hline \hline Article Info \\
\hline Article history: \\
Received Apr 29, 2019 \\
Revised Aug 12, 2019 \\
Accepted Sep 9, 2019 \\
\hline
\end{tabular}

Keywords:

Clearing-based niche formation technique Differential evolution Optimal reactive power Transmission loss Variable mesh optimization algorithm

\section{Corresponding Author:}

Kanagasabai Lenin, Department of EEE, Prasad V. Potluri Siddhartha Institute of Technology, Chalasani Nagar, Kanuru, Vijayawada, Andhra Pradesh 520007, India.

Email: gklenin@gmail.com

\begin{abstract}
In this work Improved Variable Mesh Optimization Algorithm (IVM) has been applied to solve the optimal reactive power problem. Projected Improved VMO algorithm has been modeled by hybridization of Variable mesh optimization algorithm with Clearing-Based Niche Formation Technique, Differential Evolution (DE) algorithm. Mesh formation and exploration has been enhanced by the hybridization. Amongst of niche development process, clearing is a renowned method in which general denominator is the formation of steady subpopulations (niches) at all local optima (peaks) in the exploration space. In Differential Evolution (DE) population is formed by common sampling within the stipulated smallest amount and maximum bounds. Subsequently DE travel into the iteration process where the progressions like, mutation, crossover, and selection, are followed. Proposed Improved Variable Mesh Optimization Algorithm (IVM) has been tested in standard IEEE 14,300 bus test system and simulation results show the projected algorithm reduced the real power loss extensively.
\end{abstract}

Copyright $@ 2019$ Institute of Advanced Engineering and Science. All rights reserved.

\section{INTRODUCTION}

Reactive power problem plays a key role in secure and economic operations of power system. Optimal reactive power problem has been solved by variety of types of methods [1-6]. Nevertheless, numerous scientific difficulties are found while solving problem due to an assortment of constraints. Evolutionary techniques [7-14] are applied to solve the reactive power problem, but the main problem is many algorithms get stuck in local optimal solution \& failed to balance the Exploration \& Exploitation during the search of global solution. In this work Improved Variable Mesh Optimization Algorithm (IVM) has been applied to solve the optimal reactive power problem. Projected Improved VMO algorithm has been modeled by hybridization of Variable mesh optimization algorithm with Clearing-Based Niche Formation Technique, Differential Evolution (DE) algorithm. Mesh formation and exploration has been enhanced by the hybridization. Amongst of niche development process, clearing is a renowned method in which general denominator is the formation of steady subpopulations (niches) at all local optima (peaks) in the exploration space. Each niche has a leading (master) individual, i.e. the one with the most excellent fitness. In Differential Evolution (DE) population is formed by common sampling within the stipulated smallest amount and maximum bounds. Subsequent to the launch of generating the population, DE travel into the iteration process where the progressions like, mutation, crossover, and selection, are followed. Proposed Improved Variable Mesh Optimization Algorithm (IVM) has been tested in standard IEEE 14,300 bus test system and simulation results show the projected algorithm reduced the real power loss extensively. 


\section{PROBLEM FORMULATION}

Objective of the problem is to reduce the true power loss

$$
\mathrm{F}=\mathrm{P}_{\mathrm{L}}=\sum_{\mathrm{k} \in \mathrm{Nbr}} \mathrm{g}_{\mathrm{k}}\left(\mathrm{V}_{\mathrm{i}}^{2}+\mathrm{V}_{\mathrm{j}}^{2}-2 \mathrm{~V}_{\mathrm{i}} \mathrm{V}_{\mathrm{j}} \cos \theta_{\mathrm{ij}}\right)
$$

Voltage deviation given as follows

$$
\mathrm{F}=\mathrm{P}_{\mathrm{L}}+\omega_{\mathrm{v}} \times \text { Voltage Deviation }
$$

Voltage deviation given by

$$
\text { Voltage Deviation }=\sum_{\mathrm{i}=1}^{\mathrm{Npq}}\left|\mathrm{V}_{\mathrm{i}}-1\right|
$$

Constraint (Equality)

$$
P_{G}=P_{D}+P_{L}
$$

Constraints (Inequality)

$$
\begin{aligned}
& \mathrm{P}_{\text {gslack }}^{\min } \leq \mathrm{P}_{\text {gslack }} \leq \mathrm{P}_{\text {gslack }}^{\max } \\
& \mathrm{Q}_{\mathrm{gi}}^{\min } \leq \mathrm{Q}_{\mathrm{gi}} \leq \mathrm{Q}_{\mathrm{gi}}^{\mathrm{max}}, \mathrm{i} \in \mathrm{N}_{\mathrm{g}} \\
& \mathrm{V}_{\mathrm{i}}^{\mathrm{min}} \leq \mathrm{V}_{\mathrm{i}} \leq \mathrm{V}_{\mathrm{i}}^{\max }, \mathrm{i} \in \mathrm{N} \\
& \mathrm{T}_{\mathrm{i}}^{\mathrm{min}} \leq \mathrm{T}_{\mathrm{i}} \leq \mathrm{T}_{\mathrm{i}}^{\max }, \mathrm{i} \in \mathrm{N}_{\mathrm{T}} \\
& \mathrm{Q}_{\mathrm{c}}^{\min } \leq \mathrm{Q}_{\mathrm{c}} \leq \mathrm{Q}_{\mathrm{C}}^{\max }, \mathrm{i} \in \mathrm{N}_{\mathrm{C}}
\end{aligned}
$$

\section{VARIABLE MESH OPTIMIZATION}

Variable mesh optimization algorithm (VMO) engendered population is scattered as a mesh. Mesh is poised of $\mathrm{Z}$ nodes $\left(m_{1}, m_{2}, \ldots, m_{z}\right)$ that symbolize the solutions in the search space [15]. Every node is oblique as a vector of $\mathrm{M}$ floating point numbers $m_{i}=\left(g_{1}^{i}, g_{2}^{i}, \ldots, g_{j}^{i}, \ldots, g_{i}^{M}\right)$ which designate the solution. In exploration procedure two methodologies called development and narrowing are utilized. During the development, new-fangled nodes are created in the direction of local maximum, comprehensive end and the boundary nodes. Grounded on an elite approach, nodes are prearranged bequeath to their superiority in ascending order. Then clear out adaptive operator is then applied; every node is evaluated to its successor to eradicate those that do not outdo the threshold. Threshold value is computed by

$$
\varepsilon_{j}=\left\{\begin{array}{c}
\frac{\operatorname{range}\left(k_{j}, l_{j}\right)}{4} \text { if } d<0.149 \% D \\
\frac{\text { range }\left(k_{j}, l_{j}\right)}{8} \text { if } 0.149 \% D \leq d<0.29 \% D \\
\frac{\operatorname{range}\left(k_{j}, l_{j}\right)}{16} \text { if } 0.29 \% D \leq d<0.59 \% D \\
\frac{\text { range }\left(k_{j}, l_{j}\right)}{50} \text { if } 0.59 \% D \leq d<0.79 \% d \\
\frac{\text { range }\left(k_{j}, l_{j}\right)}{100} \text { if } d \geq 0.79 \% D
\end{array}\right.
$$

Maximum number of fitness assessment fixed by D and d symbolize the present number of fitness evaluation. Range $\left(k_{j}, l_{j}\right)$ indicates the domain borders of every component. Node creation procedure at every cycle has been done.

Commence

For primary mesh randomly construct $\mathrm{Z}$ nodes

In primary mesh choose the global best

Int. J. of Adv. in Appl. Sci. Vol. 8, No. 4, December 2019: 279 - 284 
Replicate

For every node in primary mesh do

Discover adjoining $\mathrm{k}$ nodes by the spatial position

Choose the premium neighbour by fitness values

When present node is not the local most excellent then create a new-fangled node towards the local most excellent

End if

End for

For every node in primary mesh however the global most excellent do

Create a fresh node towards the global most excellent

End for

Engender nodes from the mesh boundary nodes

According to fitness values categorize the nodes

An adaptive clearing operator splodge

Choose $\mathrm{Z}$ best nodes to construct the primary mesh for the successive iterations

If essential capriciously engender new-fangled nodes to form the preliminary mesh for the subsequent iteration

When end criterion is met, process will be stopped

End

\section{CLEARING-BASED NICHE FORMATION TECHNIQUE}

Amongst of niche development process, clearing is a renowned method in which general denominator is the formation of steady subpopulations (niches) at all local optima (peaks) in the exploration space. Each niche has a leading (master) individual, i.e. the one with the most excellent fitness [16]. To a certain niche an individual fit in when its distance to the leading (master) individual is less than a given threshold called as clearing radius. This process share the possessions of a niche among a set of winners (individuals to be profited by clearing), whereas it sets to zero then the fitness of all erstwhile individuals will be in the same niche. Those restrained by the winner are deceitfully separated from the population. Subsequently reiterate this method for a definite number of iterations, then all winners will come into view.

\section{Start}

Arrange the population $P p$ in decreasing order with respect to the fitness values

For $i=0$ to $S-1$

If $($ Fitness $(P p[i]) \neq 0)$; nbWinners $=1$

Generate a new-fangled niche, being $P p[i]$ its master

For $j=i+1$ to $S-1$; if (Fitness $(P p[j]) \neq 0$ and Distance $(P p[i], P p[j])<\sigma)$

If $(n b$ Winners $<\kappa)$ nbWinners $=$ nbWinners +1

Place the individual $P p[j]$ in the present niche

Else

Fitness $(P p[j])=0$

End if

End for

End

\section{DIFFERENTIAL EVOLUTION}

In Differential Evolution (DE) population is formed by common sampling within the stipulated smallest amount and maximum bounds [17]. Subsequent to the launch of generating the population, DE travel into the iteration process where the progressions like, mutation, crossover, and selection are followed.

"DE/best/1"

$$
D_{i}=Y_{\text {best }}+H\left(Y_{s 1}-Y_{s 2}\right)
$$

"DE/current-to-best/1"

$$
D_{i}=Y_{i}+H\left(Y_{\text {best }}-Y_{i}\right)+H\left(Y_{s 1}-Y_{s 2}\right)
$$


"DE/best/2"

$$
D_{i}=Y_{\text {best }}+H\left(Y_{s 1}-Y_{s 2}\right)+H\left(Y_{s 3}-Y_{s 4}\right)
$$

"DE/rand/1"

$$
D_{i}=Y_{s 1}+H\left(Y_{s 2}-Y_{s 3}\right)
$$

"DE/current-to-rand/1"

$$
D_{i}=Y_{i}+H\left(Y_{s 1}-Y_{i}\right)+H\left(Y_{s 2}-Y_{s 3}\right)
$$

$\mathrm{DE} / \mathrm{rand} / 2 "$

$$
D_{i}=Y_{r 1}+H\left(Y_{s 2}-Y_{s 3}\right)+H\left(Y_{s 4}-Y_{s 5}\right)
$$

Improved strategy of the binomial crossover described as follows

$$
\begin{gathered}
g_{i j}=\left\{\begin{array}{c}
d_{i, j} \text { ifrand }(0,1) \leq E_{r} \text { or } l=\mathrm{l}_{\text {rand }} \\
y_{i, j} \text { otherwise }
\end{array}\right. \\
Y_{i}=\left\{\begin{array}{c}
G_{i} \text { iffitness }\left(G_{i}\right) \leq \text { fitness }\left(Y_{i}\right) \\
Y_{i} \text { otherwise }
\end{array}\right.
\end{gathered}
$$

Begin

Population are initialized

Calculate the primary population

For $\mathrm{i}=0$ to max-iteration do

Select capricious trial vectors

Create off spring's population

Combine parent and offspring population

If an offspring is greater than its parent then exchange the parent by offspring in the succeeding generation

End if

End for

End

\section{IMPROVED VARIABLE MESH OPTIMIZATION ALGORITHM}

In this work Improved Variable Mesh Optimization Algorithm (IVM) has been hybridized with Clearing-Based Niche Formation Technique, DE algorithm. Mesh formation and exploration has been enhanced through the hybridization.

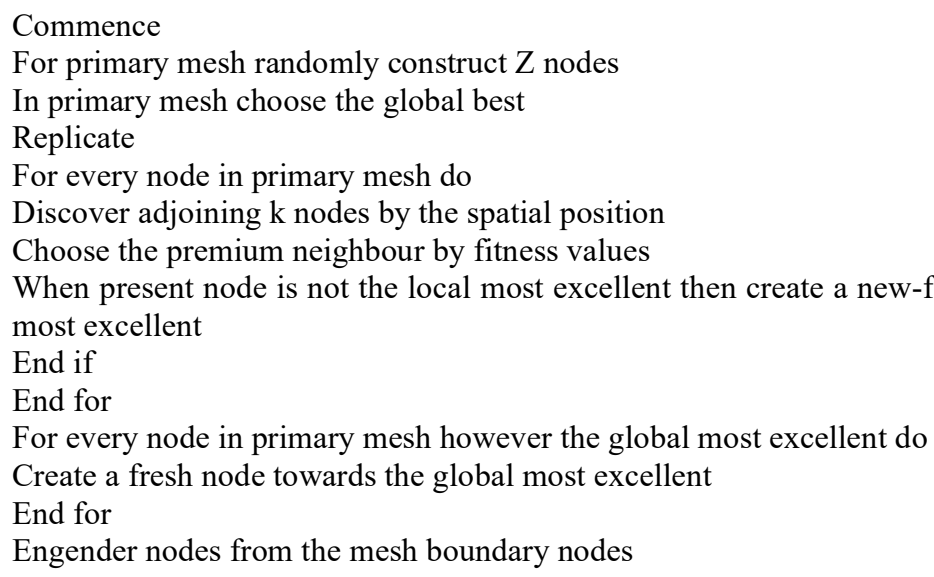

Int. J. of Adv. in Appl. Sci. Vol. 8, No. 4, December 2019: 279 - 284 
Apply the clearing-based niche formation technique

For each identified niche do

Sort nodes according to their fitness values

Apply the adaptive clearing operator

End for

According to fitness values categorize the nodes

An adaptive clearing operator splodge

Choose $\mathrm{Z}$ best nodes to construct the primary mesh for the successive iterations

If essential capriciously engender new-fangled nodes to form the preliminary mesh for the subsequent iteration

\section{DE call using VMO population}

When end criterion is met, process will be stopped

End

\section{SIMULATION RESULTS}

At first in standard IEEE 14 bus system the validity of the proposed Improved Variable Mesh Optimization Algorithm (IVM) has been tested \& comparison results are presented in Table 1.

Table 1. Improved Variable Mesh optimization algorithm (IVM)

\begin{tabular}{cccc}
\hline Control variables & $\mathrm{ABCO}[18]$ & $\mathrm{IABCO}[18]$ & IVM \\
\hline V1 & 1.06 & 1.05 & 1.02 \\
V2 & 1.03 & 1.05 & 1.03 \\
V3 & 0.98 & 1.03 & 1.01 \\
V6 & 1.05 & 1.05 & 1.00 \\
V8 & 1.00 & 1.04 & 0.90 \\
Q9 & 0.139 & 0.132 & 0.100 \\
T56 & 0.979 & 0.960 & 0.900 \\
T47 & 0.950 & 0.950 & 0.900 \\
T49 & 1.014 & 1.007 & 1.000 \\
Ploss (MW) & 5.92892 & 5.50031 & 4.0986 \\
\hline
\end{tabular}

Then IEEE 300 bus system [19] is used as test system to validate the performance of the Improved Variable Mesh Optimization Algorithm (IVM). Table 2 shows the comparison of real power loss obtained after optimization.

Table 2. Comparison of real power loss

\begin{tabular}{lllll}
\hline Parameter & Method EGA [20] & Method EEA [20] & Method CSA [21] & IVM \\
\hline PLOSS (MW) & 646.2998 & 650.6027 & 635.8942 & 613.1240 \\
\hline
\end{tabular}

\section{CONCLUSION}

In this work Improved Variable Mesh Optimization Algorithm (IVM) has been successfully solved the optimal reactive power problem. Mesh formation and exploration has been enhanced by the hybridization. Amongst of niche development process, clearing is a renowned method in which general denominator is the formation of steady subpopulations (niches) at all local optima (peaks) in the exploration space. In Differential Evolution (DE) population is formed by common sampling within the stipulated smallest amount and maximum bounds. Proposed Improved Variable Mesh Optimization Algorithm (IVM) has been tested in standard IEEE 14,300 bus test system and simulation results show the projected algorithm reduced the real power loss extensively.

\section{REFERENCES}

[1] K. Y. Lee, "Fuel-cost minimisation for both real and reactive-power dispatches," Proceedings Generation, Transmission and Distribution Conference, vol. 131(3), pp. 85-93, 1984.

[2] N. I. Deeb, "An efficient technique for reactive power dispatch using a revised linear programming approach," Electric Power System Research, vol. 15(2), pp. 121-134, 1998.

[3] M. R. Bjelogrlic, M. S. Calovic, and and B. S. Babic, "Application of Newton's optimal power flow in voltage/reactive power control," IEEE Trans Power System, vol. 5(4), pp. 1447-1454, 1990. 
[4] S. Granville, "Optimal reactive dispatch through interior point methods," IEEE Transactions on Power System, vol. 9(1), pp. 136-146, 1994.

[5] N. Grudinin, "Reactive power optimization using successive quadratic programming method," IEEE Transactions on Power System, vol. 13(4), pp. 1219-1225, 1998.

[6] Wei Yan, J. Yu, D. C. Yu, and K. Bhattarai, "A new optimal reactive power flow model in rectangular form and its solution by predictor corrector primal dual interior point method," IEEE Trans. Pwr. Syst, vol. 21(1), pp. 61-67, 2006.

[7] Aparajita Mukherjee and Vivekananda Mukherjee, "Solution of optimal reactive power dispatch by chaotic krill herd algorithm," IET Gener. Transm. Distrib, vol. 9(15), pp. 2351-2362, 2015.

[8] Z. Hu, X. Wang, and Taylor, "Stochastic optimal reactive power dispatch: Formulation and solution method," Electr. Power Energy Syst, vol. 32, pp. 615-621, 2010.

[9] M. Morgan, Nor Abdullah, Mohd Sulaiman, M. Mustafa, and Rosdiyana Samad., "Multi-objective evolutionary programming (MOEP) using mutation based on Adaptive Mutation Operator (AMO) applied for optimal reactive power dispatch," ARPN Journal of Engineering and Applied Sciences, vol. 11(14), 2016.

[10] Pandiarajan, K. \& Babulal, C. K, "Fuzzy harmony search algorithm based optimal power flow for power system security enhancement," International Journal Electric Power Energy Syst, vol. 78, pp. 72-79, 2016.

[11] Mahaletchumi Morgan, Nor Rul Hasma Abdullah, Mohd Herwan Sulaiman, Mahfuzah Mustafa, and Rosdiyana Samad, "Benchmark studies on optimal reactive power dispatch (ORPD) based multi-objective evolutionary programming (MOEP) using mutation based on adaptive mutation adapter (AMO) and polynomial mutation operator (PMO)," Journal of Electrical Systems, pp. 12-1, 2016.

[12] Rebecca Ng Shin Mei, Mohd Herwan Sulaiman, and Zuriani Mustaffa, "Ant Lion Optimizer for Optimal Reactive Power Dispatch Solution," Journal of Electrical Systems Special Issue AMPE 2015, pp. 68-74, 2016.

[13] A. Gagliano and F. Nocera, "Analysis of the performances of electric energy storage in residential applications," International Journal of Heat and Technology, vol. 35(1), pp. S41-S48, 2017.

[14] M. Caldera, P. Ungaro, G. Cammarata, and G. Puglisi, "Survey-based analysis of the electrical energy demand in Italian households," Mathematical Modelling of Engineering Problems, vol. 5(3), pp. 217-224, 2018.

[15] A. Puris, R. Bello, D. Molina, and F. Herrera, "Variable mesh optimization for continuous optimization problems," Soft Comput., vol. 16, pp. 511-525, 2018.

[16] K. Price, R. M. Storn, and J. A. Lampinen, "Differential Evolution: A Practical Approach to Global Optimization," Springer, 2006.

[17] A. Pétrowski, "A clearing procedure as a niching method for genetic algorithms," Proc. 3rd IEEE International Conference on Evolutionary Computation, Nagoya, Japan, pp. 798-803, 1996.

[18] Chandragupta Sivalingam, Subramanian Ramachandran, and Purrnimaa Shiva Sakthi Rajamani, "Reactive power optimization in a power system network through metaheuristic algorithms," Turkish Journal of Electrical Engineering \& Computer Science, vol. 25, pp. 4615-4623, 2017.

[19] IEEE, "The IEEE-test systems," 1993. [Online]: Available: http://www.ee.washington.edu/trsearch/pstca/

[20] S. S. Reddy, et al., "Faster evolutionary algorithm based optimal power flow using incremental variables," Electrical Power and Energy Systems, vol. 54, pp. 198-210, 2014.

[21] S. Surender Reddy, "Optimal reactive power scheduling using cuckoo search algorithm," International Journal of Electrical and Computer Engineering, vol. 7(5), pp. 2349-2356. 2017.

Int. J. of Adv. in Appl. Sci. Vol. 8, No. 4, December 2019: 279 - 284 